\title{
Second stage surgery: a clinical report comparing efficacy of laser and electrocautery
}

\author{
Rohit Raghavan ${ }^{1}$, Shajahan PA ${ }^{1}$, Anil Koruthu ${ }^{1}$, \\ B Sukumar ${ }^{2}$, Anoop Nair ${ }^{3}$, Divakar KP ${ }^{4} *$ \\ ${ }^{1}$ Dept. of prosthodontics, royal dental college, iron hills, chalissery, Palakkad District, Kerala, India \\ ${ }^{2}$ Dept. of prosthodontics, dental college, azamgarh, uttarpradesh, India \\ ${ }^{3}$ Dept. of prosthodontics, govt. dental college and research institute, Bangalore, Karnataka, India \\ ${ }^{4}$ Dept. of conservative dentistry and endodontics, r.v.dental college, Bangalore, Karnataka, India \\ *Corresponding author E-mail: drdivukp@gmail.com
}

\begin{abstract}
Implant exposure for impression making in second stage surgery is a very important clinical step in implant rehabilitation. It can be done in three different ways; scalpel, electrocautery and lasers. With increasing number of dentists preferring to explore the modern technology, use of electro cautery and the lasers have become popular. However the choice between an electrocautery and laser is a difficult one. This clinical report employs both the modalities in the same patient and weighs pros and cons of each of them.
\end{abstract}

Keywords: Dental implant, Electrocautery, Laser, Second stage surgery.

\section{Introduction}

In recent years the treatment options and modalities for achieving optimal functional and aesthetic outcomes with implant restorations have clearly changed (Dooren 2007).Treatment with fixed prostheses supported by endosseous implants has improved the quality of life of the edentulous patient (Cibirka et al.1997). Restoring the edentulous space with implant supported restoration involves a minimum of 3-4 clinical steps. After pre-operative analysis of the patient, in the first clinical procedure (Surgical phase) surgeon places the selected dental implant. Following the standard healing protocol, the implant is subsequently exposed in the second clinical appointment after successful osseointegration has been confirmed using the radiographs. After exposure of the implant, a healing cap/abutment is screwed on the implant and left in place for $48 \mathrm{hrs}$ until the surrounding soft tissue heals around the abutment to create an esthetic emergence profile for the final restoration. Following this clinical appointment, patient is recalled for the third phase (Impression/prosthetic phase) when the impressions for the final restorations are made. This article concentrates on the procedures that are carried out in second clinical appointment when the implant is exposed. Three methods are frequently mentioned in the literature and practiced for exposing the submerged implant. Conventional technique involves use of scalpel with operator preferred incisions to expose the implant while the modern technology uses electrosurgery and lasers to do the same. Many operators still prefer the use of scalpel as it is easily available in any simple operatory. Ease of use, low cost, relatively fast and uneventful healing are among the other reasons for the widespread continued use of scalpel. However excessive unnecessary cuts or incisions/exposure, blood at the site of surgery and impaired operatory site visibility are its disadvantages. Hence it is wise for an operator to use the modern bloodless field surgical techniques especially if the equipments are readily available in the operatory. An argument still exists regarding which method is best for soft tissue cutting electrosurgery or laser (Corbitt JD Jr 1991, Hunter 1992, Bordelon et al. 1993, Wollin \& Denstedt 1998, Boxem et al. 1999, Burns et al. 2007). The manuscript presents a case report for comparison of healing following second stage surgery in which implants where bilaterally placed in the maxilla and subsequently implant exposure done using electrosurgery and laser on contralateral sides with oral cavity acting as a split mouth model.

\section{Case report}

A 56-year-old male patient presented with a missing maxillary left canine and first premolar, right first premolar and first molar due to decay [Fig. 1]. For the missing tooth, all the treatment probabilities were discussed and patient decided to go ahead with implant placement. Titanium implant placement was done using standard protocol and patient was recalled after months for prosthetic rehabilitation.

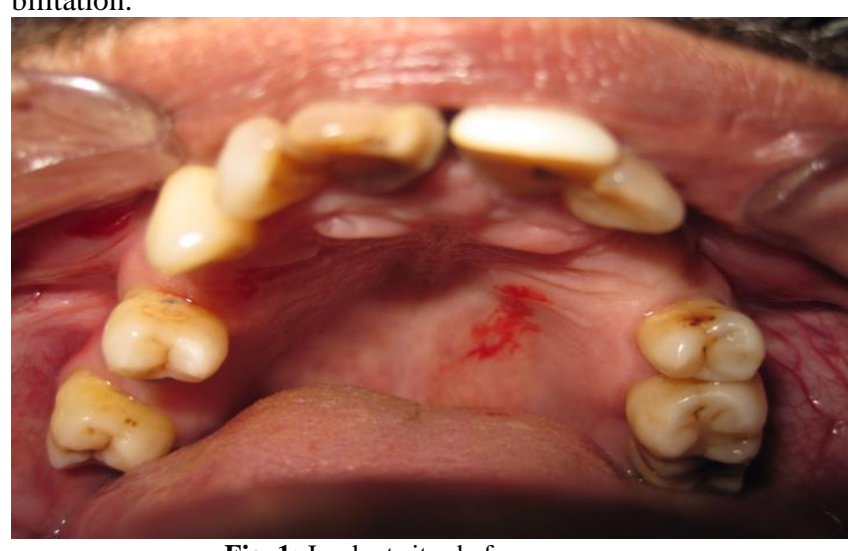

Fig. 1: Implant sites before exposure 
For the second stage surgery it was decide to expose the implant on the left side using diode laser while the right side was exposed using electrocautery [Fig. 2a. 3a].

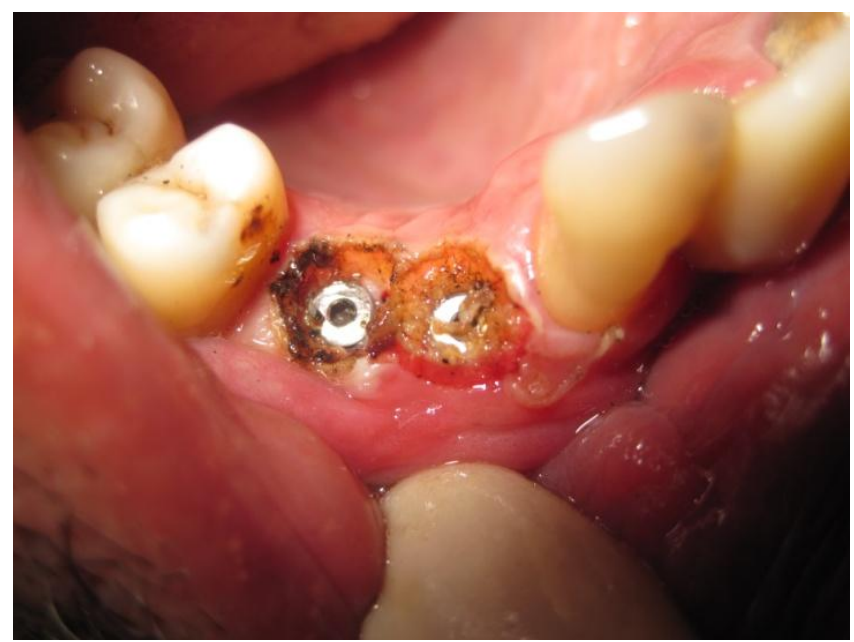

Fig. 2a: Implant sites exposed using Diode laser (Immediate post-op)

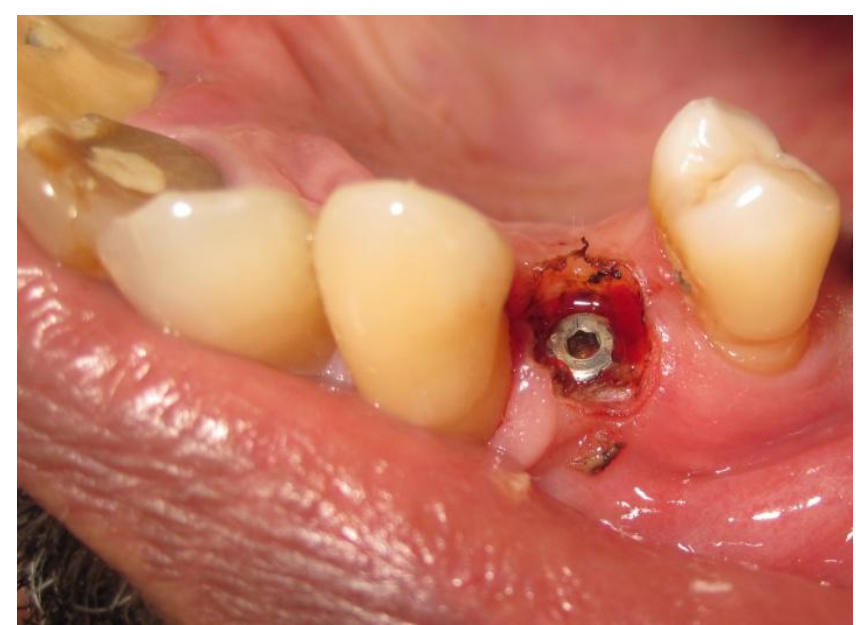

Fig. 3a: Implant sites exposed using Electrocautery (Immediate post-op)

With electrocautery it was noticed that the electrode cuts on its sides as well as on its tip. The electrode may be bent to meet the clinical need and the cuts are made with comparative ease. The hemostasis achieved is immediate, the cutting was consistent, the wound was nearly painless after the procedure and the soft tissue had minimal trauma. The healing too was very uneventful and was complete in just 7 days [Fig. 3b, 3c, 3d]. However the procedure comes with few drawbacks. Anesthetic was required for cutting; poor patient acceptance, objectionable odor and low tactile sensitivity.

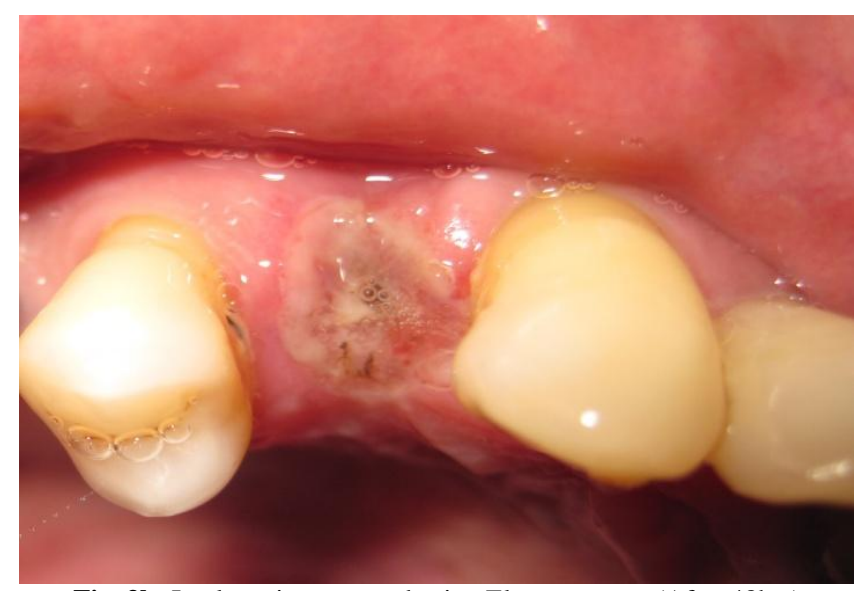

Fig. 3b: Implant sites exposed using Electrocautery (After 48hrs)

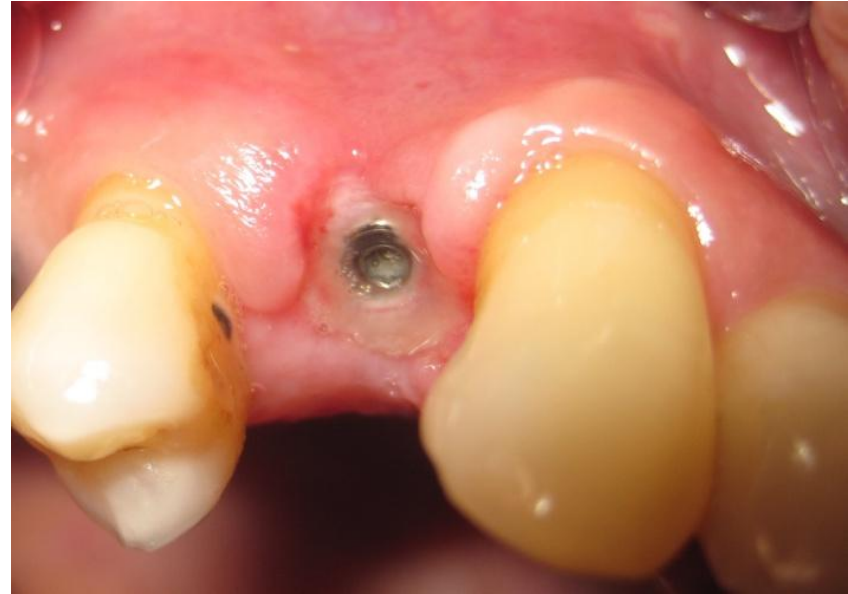

Fig. 3c: Implant sites exposed using Electrocautery (After 7 days)

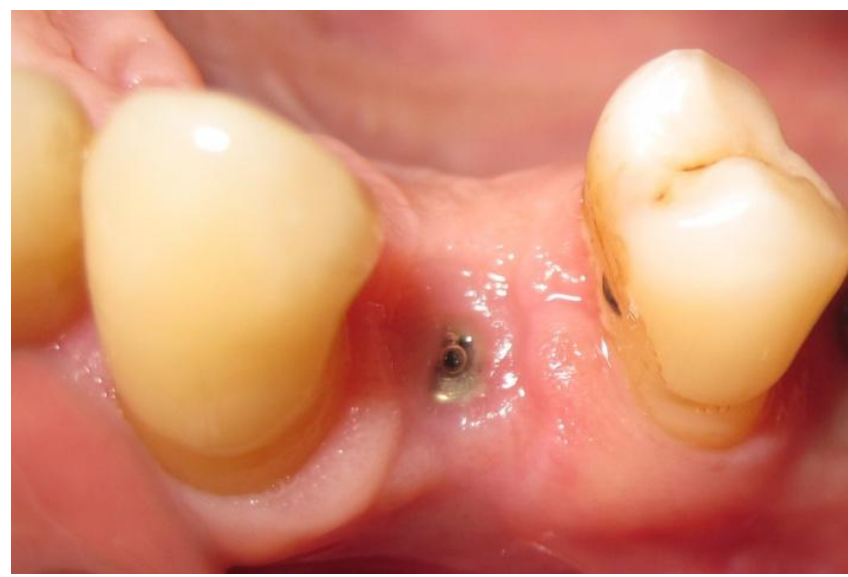

Fig. 3d: Implant sites exposed using Electrocautery (After 14 days)

With laser it was noticed that their use required minimal or no anesthetic; low or no heat production and increased patient acceptance. One of the major advantages of laser over electrocautery was that unlike electrocautery when the implant surface is touched by the working tip of laser the patient felt no shock and hence increased patient acceptance. In addition there is growing evidence that laser use may be positive therapy for periodontal disease and about its role in destroying the endotoxins and rendering the field sterile. Disadvantages include the comparatively higher cost of laser, potential hazard of laser light and laser plume, learning period and strict precautions required with lasers; slower cutting with lasers than that with electrosurgery and hence time consuming. The healing was very slow and uneventful. Complete process of healing required 3 weeks as opposed to electrosurgery [Fig. 2b, 2c, 2d].

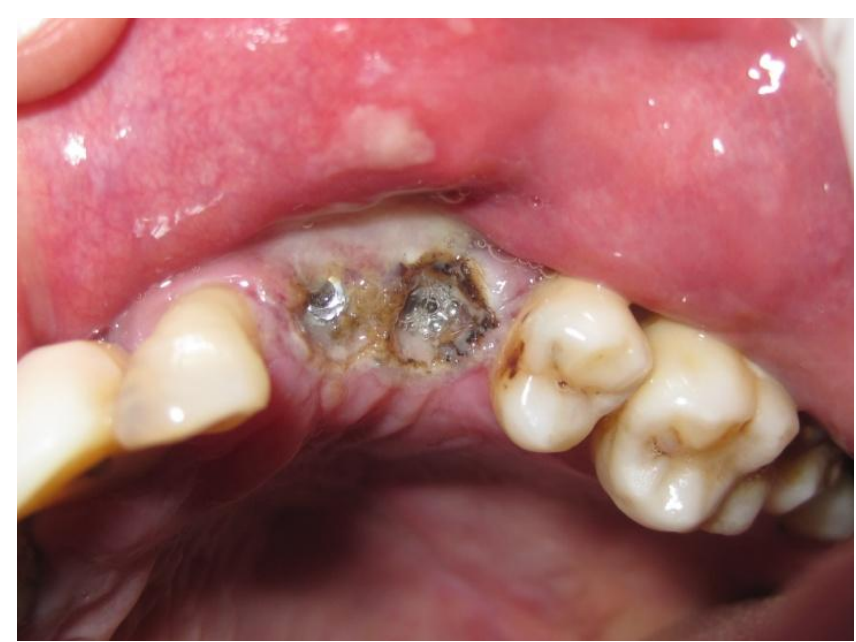

Fig. 2b: Implant sites exposed using Diode laser (After 48hrs) 


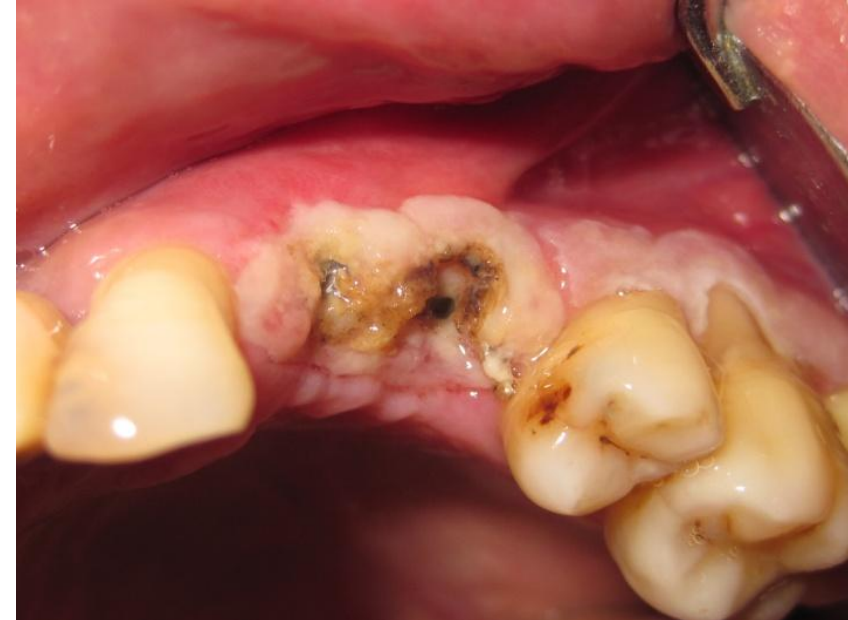

Fig. 2c: Implant sites exposed using Diode laser (After 7 days)

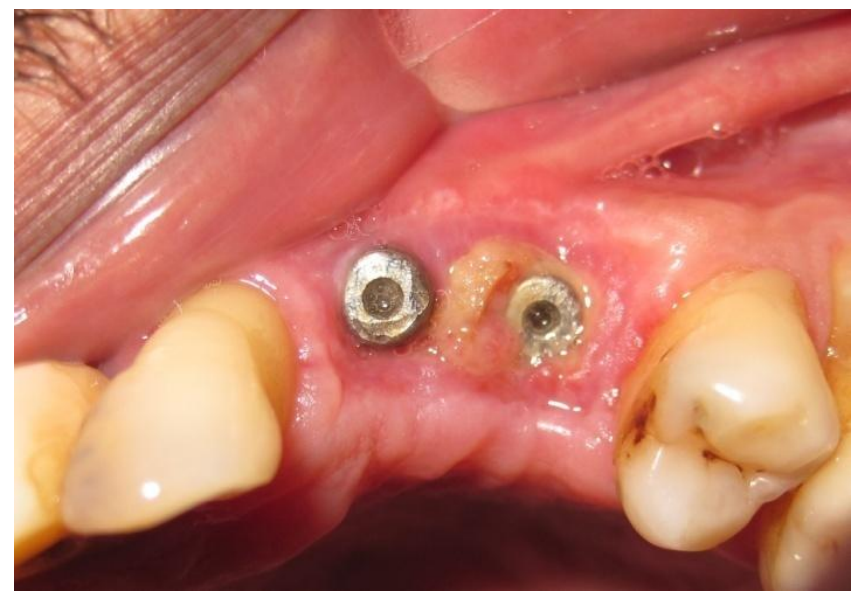

Fig. 2d: Implant sites exposed using Diode laser (After 14 days)

\section{Discussion}

Electrocautery is a technique frequently now being used as an alternative to conventional technique. Electrosurgery is a controlled, precise application of heat to the soft-tissue site to be cut, achieved by means of carefully designed electrodes. The result is a controlled, irreversible thermal alteration of the soft tissue (Christensen 2008). Laser is an acronym for light amplification by stimulated emission of radiation. Soft tissue lasers are increasing in popularity among clinicians in part due to their potential value in preprosthetic gingival procedures. The ability of soft tissue lasers to control moisture and facilitate hemostasis appears particularly promising for clinicians excising gingival tissues, performing esthetic crown lengthening, and using resective techniques for gingival troughing and these applications will grow as practitioners become more familiar with such technologies (Lee 2006). Implant exposure is one such clinical procedure that is now increasingly tried by dentists. After observing the suggested uses of laser and electrosurgery for soft-tissue cutting, we find that their suggested uses overlap considerably, and that both modes of cutting are effective. The table shows the comparative evaluation of the two modes of cutting by compiling the information from the literature and confirming it through the present case. There is another important piece of information specific to the application of these procedures in dental implant related procedures. The heat developed by monopolar electrosurgery units does not allow for their use around implants. However, careful use of bipolar electrosurgery is acceptable around implants because it produces less heat. In addition bone can be damaged with the improper use of electrocautery. Whereas with laser, there was low or no heat production and hence may be effectively used around dental implants. In addition they are considered antimicrobial as they re- move endotoxins from root surfaces and there is growing evidence that laser use may be positive therapy for periodontal disease. Use of laser, especially diode laser, continues to increase. There is no question that lasers attract patients, probably because of their significant use and the visibility of other laser related procedures. Additionally, laser cutting can be performed around implants. This is a significant advantage, because most dentists are involved at least with implant prosthodontics. However, as observed in the above report the time taken for complete healing with laser was much more than the electrocautery and the healing was uneventful with cautery.

\section{Conclusion}

Both lasers and electrosurgery units work well for simple cutting of oral soft tissues as opposed to the use of scalpel. Commercial advertisements have stimulated use of dental lasers by expressing their advantages. A comparison of diode laser with electrosurgery in the present report revealed significant overlap of potential uses and effectiveness. However lasers showed an unduly prolonged healing period post implant exposure. The decision regarding which modality to use is up to individual dentists. Are the advantages of diode laser use significant enough to compensate for the additional cost? That decision is up to the individual dentist.

\section{References}

[1] Dooren EV (2007). Using zirconia in esthetic implant restorations QDT.

[2] Cibirka RM, Razzoog M, Lang BR (1997). Critical evaluation of patient responses to dental implant therapy. J Prosthet Dent78, 574581.

[3] Corbitt JD Jr (1991). Laparoscopic cholecystecomy: laser versus electrosurgery. Surg Laparosc Endosc 1, 85-88.

[4] Hunter JG (1992). Laser use in laparoscopic surgery. Surg Clin North Am 72, 655-664.

[5] Bordelon BM, Hobday KA, Hunter JG (1993). Laser vs electrosurgery in laparoscopic cholecystecomy: a prospective randomized trial. Arch Surg 128, 233-236.

[6] Wollin TA, Denstedt JD (1998). The holmium laser in urology. J Clin Laser Med Surg 16, 13-20.

[7] Boxem T, Muller M, Venmans B, Postmus P, Sutedja T (1999). NdYAG laser vs bronchoscopic electrocautery for palliation of symptomatic airway obstruction: a cost-effectiveness study. Chest 116, 11081112 .

[8] Burns JA, Kobler JB, Heaton JT, Lopez- Guerra G, Anderson RR, Zeitels SM (2007). Thermal damage during thulium laser dissection of laryngeal soft tissue is reduced with air cooling: ex vivo calf model study. Ann Otol Rhinol Laryngol 116,853-857.

[9] Christensen GJ (2008). Soft-tissue cutting with laser versus electrosurgery. J Am Dent Assoc.139, 981-984.

[10]Lee EA (2006). Laser-Assisted Gingival Tissue Procedures in Esthetic Dentistry. Pract Proced Aesthet Dent. 18, suppl 2-6. 